\title{
Mamíferos pequeños no voladores del Parque Natural Municipal Ranchería, Paipa, Boyacá, Colombia
}

\section{Small nonflying mammals of Parque Natural Municipal Ranchería, Paipa, Boyacá, Colombia}

\author{
Ángela del Pilar Vianchá Sánchez ${ }^{1}$, Jarly Yalicce Cepeda-Gómez, \\ Edicka Constanza Muñoz López ${ }^{1}$, Ángela María Hernández Ochoa ${ }^{1}$, \\ Liliana Rosero Lasprilla ${ }^{2}$
}

\begin{abstract}
Resumen
Se presenta un inventario de pequeños mamíferos no voladores para el Parque Natural Municipal Ranchería (noreste del municipio de Paipa), ubicado entre los 2700 y los $3550 \mathrm{~m}$ de altitud. Este inventario es el más completo realizado hasta la fecha para el departamento de Boyacá. Los datos analizados fueron obtenidos de dos fases de campo, la fase uno realizada entre octubre 2005 a marzo 2006 y la fase dos entre enero 2007 a julio 2007. Se empleó la técnica de captura directa utilizando trampas Sherman, de golpe y de caída. Se capturaron 50 individuos en la primera fase con un esfuerzo de captura de 4320 trampas/noche y una efectividad de 1.2\%. En la segunda fase se capturaron 375 individuos, con un esfuerzo de captura de 7200 trampas/noche y una efectividad de $5.2 \%$. En total se registraron doce especies distribuidas en cuatro órdenes: Marmosa (sensu lato) (Orden Didelphimorphia), Caenolestes fuliginosus (Orden Paucituberculata), Cryptotis thomasi (Orden Soricomorpha), y las especies más abundantes de este estudio: Akodon bogotensis, Chilomys instans, Microryzomys minutus, Nephelomys aff. albigularis, Oligoryzomys fulvescens, Rhipidomys fulviventer, Thomasomys aureus, Thomasomys laniger y Thomasomys niveipes del orden Rodentia. El número de especies reportadas para el Parque, es el esperado de acuerdo con trabajos realizados para la Cordillera Oriental colombiana. Constituyen registros a tener en cuenta en los planes de manejo y conservación del área del Parque, la presencia de Thomasomys niveipes categorizada como endémica, Thomasomys aureus considerada bioindicadora de ecosistemas altoandinos y Chilomys instans especie rara.
\end{abstract}

Palabras clave: Rodentia; Cordillera Oriental colombiana; Éxito de captura.

\begin{abstract}
This paper presents the first list of non-volant small mammals of Paipa Rancheria Municipal Natural Park (PRMNP) as well as the most complete register of its kind to the date for Boyacá Departament in Colombia. PRMNP is located at the north-east of Paipa's municipality and between 2700 and $3550 \mathrm{~m}$ of altitude. The data analyzed was collected in two experimental phases. Phase one between October 2005-March 2006 and phase two, between January-July 2007. Direct capture techniques by Sherman, snap and fall traps were used. In phase one, 50 individuals were captured with a capture effort of 4320 traps/night and $1.2 \%$ index of effectiveness. In phase two 375 individuals were captured with a captured effort of 7200 traps/night and a $5.2 \%$ index of effectiveness. 12 species were registered, distributed in four orders: Marmosa (sensu lato) (Order Didelphimorphia), Caenolestes fuliginosus (Order Paucituberculata), Cryptotis thomasi (Order Soricomorpha), and belonging to Rodentia, the most abundant in this study: Akodon bogotensis, Chilomys instans, Microryzomys minutus, Nephelomys aff. albigularis, Oligoryzomys fulvescens, Rhipidomys fulviventer, Thomasomys aureus, Thomasomys laniger and Thomasomys niveipes. The species found in the Park are in expected with data reported in other papers dealing with non-volant small mammal populations in Andes mountain range (eastern cordille-
\end{abstract}

1. Bióloga, Grupo de Investigación Biología para la Conservación. Universidad Pedagógica y Tecnológica de Colombia, Tunja, Colombia.e-mail: anyiviancha@gmail.com yalicepedag@gmail.com cony9@hotmail.com angelamariastart@yahoo.com

2. Profesora Asociada, Escuela de Ciencias Biológicas, Universidad Pedagógica y Tecnológica de Colombia, Tunja, Colombia. e-mail: lilianaroseros@gmail.com

Fecha recepción: Febrero 17, $2012 \quad$ Fecha aprobación: Marzo 8, 2012 
ra). However, it may be possible to find in PRMNP species different from the ones here reported if other techniques of captured are used. The presence of the endemic species, $T$. niveipes and the rare species $C$. instans and $T$. aureus, might be considered the most important information that should be taken in to consideration, regarding the management and conservation plans for the Park.

Keywords: Rodentia; Colombian eastern cordillera; Capture success.

\section{Introducción}

Gran porcentaje de los mamíferos de Colombia se distribuyen exclusivamente en la región andina, en áreas de piedemonte, en páramos y en selva andina (Myers 1988). La zona andina cuenta con condiciones topográficas complejas y biomas con distribuciones diversas que en conjunto permiten que se desarrollen tipos de ecosistemas y especies tanto de fauna como de flora únicas en estas áreas biogeográficas.

La mayor diversidad de mamíferos en el país se da principalmente en las cordilleras Oriental y Occidental con cinco a siete especies endémicas (Rodríguez-Mahecha et al. 2006). No obstante la vertiente oriental es un área insuficientemente explorada desde el punto de vista zoológico, por ello la necesidad de incrementar los trabajos de inventarios y colecciones (Hernández 1993), que ayuden a complementar el conocimiento de la biodiversidad en el Neotrópico (Voss y Emmons 1996).

Algunos trabajos realizados de pequeños mamíferos no voladores han tratado aspectos ecológicos. Zúñiga et al. (1990) que realizaron un estudio en dos bosques cercanos a la sabana de Bogotá, en el Cerro de Majuy y Cueva Grande, encontrando mayor efectividad de muestreo y riqueza de especies en Cueva Grande que en el Cerro Majuy. López-Arévalo y Montenegro-Díaz (1993) presentan un inventario y analizan aspectos ecológicos y poblacionales. Cadena y Malagón (1994) realizaron un estudio acerca de parámetros poblaciones en el Cerro de Monserrate (Cundinamarca). Gómez-Laverde (1994) reportó 12 especies en Ucumarí, Risaralda, analizando algunos parámetros de comunidades y poblaciones. GómezValencia (2004) realizó un estudio de comunidades comparando éxitos de captura en diferentes rangos altitudinales en la Reserva Río Blanco, Manizales.
Sánchez et al. (2004) realizaron un inventario en bosque andino y altoandino de los andes centrales utilizando diferentes métodos de captura. Para el departamento de Boyacá los trabajos con este grupo de mamíferos son poco representativos, solo existen algunas series colectadas y depositadas en museos como el Field Museum Natural History (FMNH) y colecciones nacionales como la colección de mamíferos del Instituto de Ciencias Naturales (ICN) y el Instituto Alexander von Humboldt (IAvH).

La condición de la zona andina, es particularmente crítica donde son extensas la deforestación y la fragmentación del hábitat (Duran y Kattan 2005, Etter et al. 2006); se estima que se han perdido más del $74 \%$ de la cobertura forestal, lo que constituye un problema para la biodiversidad presente (Cujar 2005), por tanto la información que se adquiera con el inventario de pequeños mamíferos no voladores puede ayudar a incrementar el conocimiento actual de la biodiversidad. Por todo lo anterior, el objetivo del presente trabajo es hacer una contribución al conocimiento de las especies de mamíferos de Boyacá y del Parque Natural Municipal Ranchería con el fin de lograr propuestas para futuros trabajos que puedan ayudar a la conservación del Parque.

\section{Materiales y métodos}

Área de estudio. El estudio se realizó en el Parque Natural Municipal Ranchería, ubicado en la parte centro-oriental de Colombia y noroccidental del departamento de Boyacá en el municipio de Paipa, uno de los valles interandinos de origen aluvial de la Cordillera Oriental (Figura 1). El parque presenta un sistema montañoso entre un rango altitudinal que va desde los 2600 hasta los $3500 \mathrm{~m}$ (POT 2000), ubicado entre las coordenadas $6^{\circ} 0$ ' 25.71' y

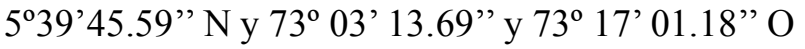
(Corpoica 2006, Barrera y Vélez 2005). Cuenta con una extensión de 679 ha, y comprende las veredas Rincón de Españoles, Jazminal y Los Medios (Barrera y Vélez 2005). El Parque se delimita para la administración, manejo, conservación y protección del ambiente, de los recursos naturales (Acuerdo 034 del 14 de diciembre del 2004 del Concejo Municipal de Paipa, Boyacá). Se caracteriza por tener formaciones vegetales de bosque altoandino y subpáramo. En el año 2005, se declara como zona 
amortiguación a 31 predios aledaños al Parque y se encuentran localizados en las veredas Los Medios, el Jazminal y Peña Amarilla (Corpoica 2006, Barrera y Vélez 2005). Presenta una vegetación propia de bosque altoandino entre $\operatorname{los} 2300$ a $3300 \mathrm{~m}$, (Cuatrecasas 1958), y según las zonas de vida de Holdridge (1967) es un bosque montano húmedo (bh$\mathrm{M})$, con nubosidad y nieblas frecuentes. El tipo de vegetación es típica de bosque altoandino con los géneros más representativos cómo Weinmannia, Brunellia, Clusia, Befaria, Drimys, Ternstroemia, Geissanthus, Daphnosis, Miconia, Oreopanax, Vallea, Eugenia, Gaiadendron, Palicourea, Tournefotia, Piper, Bocconia, Escallonia, Berberis, Symplocos, Duranta, Clethra, Rhamnus, Alnus, Ilex, Nectandra, Podocarpus, Hesperomeles y Ceroxylon (López y Monroy 2003), además el estrato epifito en general es exuberante y conspicuo. El ecosistema de subpáramo, se compone de vegetación arbustiva y matorrales, con especies como Diplostephium, Pentacalia, Gynoxis, Hypericum, Pernettya, Vaccinum y Gaultheria, conformando mosaicos y ecotonos entre un ecosistema y otro (Pardo 2007). Presenta un régimen de lluvias de tipo bimodal tetraestacional, según la información obtenida en la estación La Rusia desde los años de 1967 hasta el 2007, localizada en el municipio de Duitama (IDEAM 2008). El promedio de precipitación anual promedio es de $158.6 \mathrm{~mm}$, con dos períodos de mayores lluvias, el primero entre los meses de marzo a mayo y el segundo de septiembre a noviembre, y un período de sequía o menor proporción de lluvias en los meses de junio a agosto y de diciembre a febrero. La temperatura media anual es de $12.1^{\circ} \mathrm{C}$, con una temperatura mínima promedio de $11.08^{\circ} \mathrm{C}$ y una máxima promedio de $13.4^{\circ} \mathrm{C}$ (Figura 1).

Los muestreos se realizaron en las veredas Los Medios en los sectores: Quebrada El Olvido, Quebrada Hoyo del Infiernito y La Pradera; Rincón de Españoles en los sitios: El Pantano y Camino Pedro Hernández; y la vereda Peña Amarilla en el sector conocido como Peña Flor, sitios característicos de bosque altoandino y subpáramo.

Para la captura de pequeños mamíferos no voladores (PMNV) se utilizó la técnica de captura directa desarrollada en dos fases: la primera se realizó mensualmente de octubre 2005 a marzo 2006 y la segunda de enero 2007 a junio 2007, con salidas mensuales y con una duración de seis días durante cada fase.

Fase uno. Se realizó entre octubre 2005 y marzo 2006; se utilizaron 35 trampas Sherman de dos tamaños 10.2 x 11.4 x $38 \mathrm{~cm}$ y $7.6 \times 8.9 \times 22.5 \mathrm{~cm}$; 41 trampas de caída que consistían en recipientes de pintura limpias de cuatro litros de capacidad (diámetro, $20 \mathrm{~cm}$; altura, $28 \mathrm{~cm}$ ) enterradas a nivel del piso y 48 trampas de golpe, para un total de 120 tram-

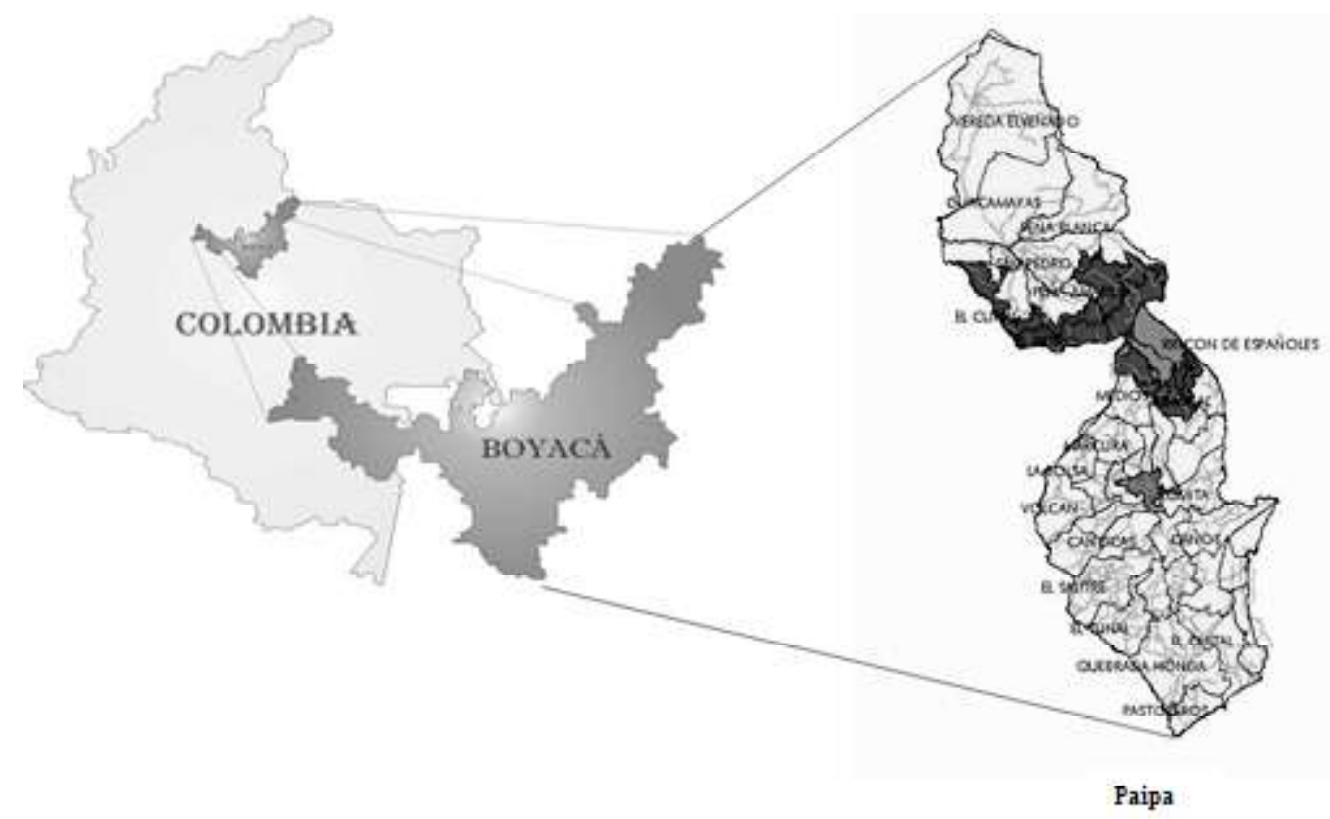

Figura 1. Área de estudio del Parque Natural Municipal Ranchería Paipa, Boyacá, Colombia. 
pas, separadas una de otra por una distancia mínima de $5 \mathrm{~m}$.

Fase dos. Se realizó entre enero y junio de 2007; se instalaron 40 trampas de golpe y 40 de caída en tres sitios simultáneos, para un total de 240 trampas por noche. Las trampas de caída, consistieron en canecas plásticas de $36.2 \mathrm{~cm}$ de profundidad instaladas a nivel del suelo.

Para ambas fases las trampas instaladas permanecieron activas durante las 24 horas de los 6 días de muestreo de cada mes. El cebo utilizado para las trampas de golpe fue una mezcla de maní, esencia de banano, avena y grasa animal (modificado de Beer 1964). Los individuos capturados se procesaron como material científico de referencia. Las carcazas se fijaron en formol al 10\% y luego se preservaron en etanol al 70\%. Los cráneos se depositaron provisionalmente en frascos con etanol al $70 \%$ para posterior limpieza por Dermestes. Cada uno de los especímenes se etiquetó con la sigla del colector y el número de colección correspondiente. A todos los individuos capturados se les tomaron las medidas convencionales para el estudio de mamíferos (Nagorsen y Peterson 1980). Los ejemplares fueron identificados por Marcela Gómez Laverde y están en proceso de ingreso a la colección de mastozoología del Instituto de Ciencias Naturales de la Universidad Nacional de Colombia o al Instituto von Humboldt de Colombia.

También se realizó una categorización de las especies capturadas en pequeñas, medianas y grandes de acuerdo con la longitud cabeza-cuerpo (se obtiene restando a la longitud total, la longitud de la cola), con el fin de proporcionar una herramienta más a tener en cuenta para su posterior identificación. Se consideraron como especies pequeñas aquellos individuos con longitud cabeza-cuerpo, con tamaños entre 75.5 mm-106 mm, especies medianas con valores entre $107.5 \mathrm{~mm}-138.5 \mathrm{~mm}$ y grandes con longitud entre $139.5 \mathrm{~mm}-172.5 \mathrm{~mm}$ (Tabla 1). La longitud de la cola es una variable que cambia de acuerdo a la especie, se categorizó en cola pequeña, cola mediana y cola larga, para los tres tamaños ya establecidos a los individuos de las especies del Parque Natural Municipal Ranchería respecto a la longitud cabeza-cuerpo que presentaron.

\section{Resultados}

Fase uno. Se capturaron 50 individuos en un total de 36 noches, con un esfuerzo de 4320 trampas/noche y un porcentaje de efectividad de trampeo de $1.2 \%$. En la Tabla 2 se muestra el número de capturas por cada tipo de trampa.

Fase dos. Se capturaron 375 individuos en un total de 30 noches, con un esfuerzo de 7200 trampas/noche y una efectividad de trampeo de 5.2\% (Tabla 3). La Tabla 4, muestra el total de los individuos capturados con las medidas morfológicas, promedios y rangos, datos obtenidos en conjunto de las fases uno y dos. Reuniendo el conjunto de datos obtenidos en las fases uno y dos, se capturaron 12 es-

Tabla 1. Especies pequeñas, medianas y grandes según tamaño y categorización de su cola del Parque Natural Municipal Ranchería, Paipa, Boyacá

\begin{tabular}{|c|c|c|c|}
\hline Especies pequeñas (75.5-106.5 mm) & $\begin{array}{c}\text { Cola pequeña (mm) } \\
(29-63)\end{array}$ & $\begin{array}{c}\text { Cola mediana }(\mathrm{mm}) \\
(64-98)\end{array}$ & $\begin{array}{c}\text { Cola larga }(\mathbf{m m}) \\
(99-133)\end{array}$ \\
\hline Cryptotis thomasi & $\mathrm{x}$ & & \\
\hline Akodon bogotensis & & $x$ & \\
\hline Chilomys instans & & & $x$ \\
\hline Microryzomys minutus & & & $x$ \\
\hline Oligoryzomys fulvescens & & $\mathrm{x}$ & \\
\hline Especies medianas $(107.5-138.5 \mathrm{~mm})$ & $(120-134)$ & $(135-149)$ & $(150-164)$ \\
\hline Caenolestes fuliginosus & $x$ & & \\
\hline Rhipidomys fulviventer & & $x$ & \\
\hline Nephelomys gr. albigularis & & & $x$ \\
\hline Thomasomys laniger & $x$ & & \\
\hline Thomasomys niveipes & & $x$ & \\
\hline Especie grande (139.5-172.5 mm) & & & $(225)$ \\
\hline Thomasomys aureus & & & $x$ \\
\hline
\end{tabular}


Tabla 2. Número de individuos de cada una de las especies de pequeños mamíferos no voladores capturados en la fase uno (octubre de 2005 a marzo de 2006) en el del Parque Natural Municipal Ranchería, Paipa, Boyacá

\begin{tabular}{lrcc}
\hline \multirow{2}{*}{ Especies } & \multicolumn{3}{c}{ Trampas } \\
\cline { 2 - 4 } & Sherman & Golpe & Caída \\
\hline Caenolestes fuliginosus & 1 & 2 & 0 \\
Cryptotis thomasi & 0 & 0 & 7 \\
Akodon bogotensis & 0 & 1 & 0 \\
Nephelomys gr. albigularis & 7 & 3 & 0 \\
Thomasomys laniger & 2 & 5 & 0 \\
Thomasomys niveipes & 13 & 9 & 0 \\
Total & 23 & 20 & 7 \\
\hline
\end{tabular}

pecies de pequeños mamíferos no voladores pertenecientes a cuatro órdenes: Didelphimorphia, Paucituberculata, Soricomorpha y Rodentia; de este último se capturó el mayor número de especies e individuos (Tabla 3).

\section{Discusión}

Las diferencias en el porcentaje de efectividad obtenidas en la fase uno (1.2\%) y la fase dos $(5.2 \%)$, probablemente se deben al tipo y cantidad de trampas empleadas, porque las trampas de caída utiliza-
Tabla 3. Número de individuos de cada una de las especies de pequeños mamíferos no voladores capturados en la fase dos (enero a julio de 2007) en el Parque Natural Municipal Ranchería, Paipa, Boyacá

\begin{tabular}{lrr}
\hline \multirow{2}{*}{ Especies } & \multicolumn{2}{c}{ Trampa } \\
\cline { 2 - 3 } & Golpe & Caída \\
\hline Marmosa (sensu lato) & 0 & 2 \\
Caenolestes fuliginosus & 24 & 20 \\
Cryptotis thomasi & 4 & 24 \\
Akodon bogotensis & 12 & 8 \\
Chilomys instans & 2 & 9 \\
Microryzomys minutus & 1 & 87 \\
Nephelomys gr. albigularis & 9 & 1 \\
Oligoryzomys fulvescens & 1 & 1 \\
Rhipidomys fulviventer & 0 & 3 \\
Thomasomys aureus & 4 & 1 \\
Thomasomys laniger & 37 & 44 \\
Thomasomys niveipes & 41 & 38 \\
Total & 135 & 238 \\
\hline
\end{tabular}

das en la fase uno eran de aluminio y de menor tamaño, mientras que las utilizadas en la fase dos eran plásticas y grandes. Además otros factores que podrían estar asociados son las épocas climáticas y experiencias de los colectores, como lo señala Gómez-Laverde (1994). El porcentaje de efectividad obtenido para la fase dos coincidió con el registrado (5.2\%) en el trabajo de Malagón (1988) y es

Tabla 4. Medidas corporales de las especies de pequeños mamíferos no voladores capturados en el Parque Natural Municipal Ranchería, Paipa, Boyacá

\begin{tabular}{|c|c|c|c|c|c|}
\hline \multirow[b]{2}{*}{ Taxón } & \multicolumn{4}{|c|}{ Medidas corporales } & \multirow[b]{2}{*}{ LO } \\
\hline & Peso (g) & LC-C & LC & LP & \\
\hline \multicolumn{6}{|l|}{ Didelphimorphia } \\
\hline Marmosa (sensu lato) $n=2$ & $27.5(21-33)$ & $102.5(100-105)$ & $136(130-142)$ & 16 & $16.5(15-16.5)$ \\
\hline \multicolumn{6}{|l|}{ Paucituberculata } \\
\hline Caenolestes fuliginosus $n=52$ & $28(13-45)$ & $107.7(62-136)$ & $126.2(110-188)$ & $22.7(21-27)$ & $11.7(7-18)$ \\
\hline \multicolumn{6}{|l|}{ Soricomorpha } \\
\hline \multicolumn{6}{|l|}{ Rodentia } \\
\hline Akodon bogotensis $\mathrm{n}=21$ & $14.1(7-18)$ & $82.1(48-112)$ & $62.2(50-73)$ & $12(7-18)$ & $18(12-20)$ \\
\hline Chilomys instans $n=11$ & $18(11-22.5)$ & $91.2(67-115)$ & $131.8(108-160)$ & $24.1(22-26)$ & $14.1(10-17)$ \\
\hline Microryzomys minutus $\mathrm{n}=79$ & $13.9(6-31)$ & $77.2(49-92)$ & $114.3(80-135)$ & $21.6(15-25)$ & $12(7-23)$ \\
\hline Nephelomys gr. albigularis $\mathrm{n}=20$ & $70.9(39-100)$ & $134.3(65-150)$ & $162.9(131-182)$ & $34(30-37)$ & $20.5(17-25)$ \\
\hline Oligoryzomys fulvescens $n=2$ & $12.2(10.5-14)$ & $75.5(71-80)$ & $96.5(93-100)$ & $20.5(21-20)$ & 18 \\
\hline Rhipidomys fulviventer $\mathrm{n}=3$ & $38.5(23.3-53.7)$ & $108(96-125)$ & $147.3(126-165)$ & $23.3(21-27)$ & $13.3(10-16)$ \\
\hline Thomasomys aureus $n=5$ & $109.6(83-140)$ & $172.4(151-194)$ & $225.4(194-243)$ & $37.2(35.5-38.9)$ & $24.8(27.9-21.7)$ \\
\hline Thomasomys laniger $\mathrm{n}=80$ & $31.3(18-45)$ & $106.8(58-172)$ & $120.2(102-170)$ & $24.7(15-36)$ & $16.7(15-36)$ \\
\hline Thomasomys niveipes $\mathrm{n}=94$ & $38.1(14-51)$ & $113.3(46-160)$ & $140.4(113-180)$ & $28.1(21-34)$ & $18.9(7-26)$ \\
\hline
\end{tabular}

Los valores corresponden al valor promedio; entre paréntesis se indica el rango de cada variable.

LC-C: Largo cabeza-cola; LC: Largo cola; LP: Largo pata; LO: Largo oreja. 
mayor que el registrado de $4.3 \%$ por MontenegroDíaz y López-Arévalo (1990) para pequeños mamíferos no voladores en la Cordillera Oriental colombiana.

Por otra parte Voss y Emmons (1996) y Voss et al. (2001), mencionan que es necesario el empleo de distintos tipos de trampas para la eficiencia en el trampeo, esto se pudo corroborar con los resultados del presente estudio, sobre todo en la fase dos, en donde se presentó una mayor eficiencia para algunos taxones utilizando un determinado tipo de trampa como para otros no. Por ejemplo, la mayoría de especímenes de $M$. minutus y $C$. thomasi fueron capturados con las trampas de caída, mientras que para $N$. albigularis hubo mayor número individuos capturados con trampas de golpe. También hubo especies como T. niveipes en las cuales la proporción de individuos capturados fue similar utilizando trampas de golpe o de caída.

Las capturas registradas en el presente inventario se encuentra en lo esperado para estudios de los Andes (Gómez-Valencia 2004); los datos obtenidos se pueden comparar con el trabajo realizado por López-Arévalo y Montenegro-Díaz (1993), quienes reportan un total de catorce especies para la Reserva Biológica Carpanta, Cundinamarca, de las cuales nueve son las mismas especies que se capturaron en este estudio (C. thomasi, A. bogotensis, C. instans, M. minutus, $N$. albigularis, $O$. fulvescens, $T$. aureus, $T$. laniger y $T$. niveipes), otras tres corresponden a Didelphis albiventris, Mustela frenata y Oryzomys sp.

Asimismo para la Cordillera Oriental el trabajo de Cadena y Malagón (1994) reportaron cuatro especies de pequeños mamíferos no voladores, las cuales tres de ellas se comparten con el actual trabajo (C. thomasi, A. bogotensis y T. laniger); Oryzomys alfaroi no se registró en el presente estudio. En la Cordillera Central, Gómez-Valencia (2004) reporta 15 especies de las cuales cinco se comparten con este estudio (C. fuliginosus, C. instans, M. minutus, $N$. albigularis y T. aureus). En general el estudio que más se acerca a los resultados obtenidos es el de López-Arévalo y Montenegro-Díaz (1993) probablemente por la proximidad geográfica.

Con base en la agrupación por tamaños de las especies capturadas (cinco pequeñas, cinco medianas y una grande) el presente trabajo difiere de lo reportado por Gómez-Valencia (2004) quien presenta en su mayoría especies pequeñas, seguidas de unas pocas medianas y una grande.

El orden Rodentia fue el más abundante en cuanto al número de especies y especímenes registrados en comparación con los demás órdenes registrados en este estudio, esto concuerda con otros trabajos en los cuales se ha hecho inventarios de PMNV en el país.

Notablemente en varios trabajos de PMNV se ha reportado $A$. bogotensis como endémica para Colombia como lo señala Albérico et al. (2000); sin embargo, es necesario aclarar que en el trabajo de Wilson y Reeder (2005) consideran a esta especie con una distribución que incluye las Cordilleras de los Andes de Venezuela. Esta especie se asocia con bosque nublado entre los 2360 y 3850 m, muestra una adaptación a un hábitat semifosorial que le permite desplazarse con mayor facilidad en el páramo (López-Arévalo y Montenegro-Díaz 1993).

Por otro lado hay que mencionar que $C$. instans, es considerada según López-Arévalo y MontenegroDíaz (1993), Gómez-Laverde (1994) y Gómez-Valencia (2004), como una especie rara y en el presente estudio también se presentaron bajas capturas. De igual forma la presencia de las especies $R$. fulviventer, T. aureus y $O$. fulvescens, con bajas capturas se podrían denominar «raras»; sus tasas bajas de captura probablemente estarían relacionadas con la época de muestreo y las técnicas de capturas utilizadas porque $R$. fulviventer y $T$. aureus son especies arborícolas (Montenegro-Díaz y López-Arévalo 1990), además el bajo número de capturas se podría deber a las poblaciones muy reducidas de estos.

Por otro lado hay que mencionar que $C$. instans, es considerada según López-Arévalo y MontenegroDíaz (1993), Gómez-Laverde (1994) y Gómez-Valencia (2004), como una especie rara; en el presente estudio también se presentaron capturas bajas. De igual forma la presencia de las especies $R$. fulviventer, T. aureus, y $O$. fulvescens, con capturas bajas se podrían denominar «raras»; sus tasas bajas de captura probablemente estarían relacionadas con la época de muestreo y las técnicas de capturas utilizadas porque $R$. fulviventer y $T$. aureus son especies arborícolas (Montenegro-Díaz y López-Arévalo 1990).

El registro de $T$. niveipes, categorizada como endémica (Albérico et al. 2000), constituye un registro muy importante. Esta especie es similar a $T$. 
laniger. Una característica que diferencia a estas dos especies es la coloración de su pata: más oscura en T. laniger y más clara en T. niveipes (Gómez-Laverde et al. 1997). Además $T$. laniger se sitúa más hacia los bosques y $T$. niveipes se mantiene en ecosistemas de páramo restringido en los departamentos de Cundinamarca y Boyacá a elevaciones por encima de los 2900 m (Gómez-Laverde et al. 1997). C. fuliginosus, C. thomasi, M. minutus y N. albigularis, son especies que se pueden considerar comunes en las capturas de mamíferos pequeños no voladores en el área del Parque.

El presente estudio muestra que los resultados del inventario se encuentran dentro de lo esperado en términos de capturas de pequeños mamíferos no voladores; de igual forma se presume la presencia de otras especies de pequeños mamíferos no voladores. Es de esperar la presencia de este mismo número de especies para todo el departamento de Boyacá en ecosistemas altoandinos; sin embargo, la presencia de estas especies dependerá del grado de conservación en el que se encuentre el ecosistema. También ayuda a complementar la información de mamíferos para la Cordillera Oriental colombiana e incentiva a proponer herramientas para la conservación de estas áreas naturales protegidas y otras aún no exploradas del departamento de Boyacá.

\section{Agradecimientos}

A la Corporación Autónoma y Regional de Boyacá (CORPOY ACA) y la Dirección de Investigación de la Universidad Pedagógica y Tecnológica de Colombia por el financiamiento de este proyecto. A Marcela Gómez Laverde por la ayuda en la identificación de las especies colectadas y por toda su colaboración en la realización de este proyecto. A Olga Lucía Montenegro del Instituto de Ciencias Naturales de la Universidad Nacional de Colombia por la asesoría en métodos de captura de ejemplares de PMNV y colaboración para este estudio. A Catalina Fuentes por la asesoría en métodos de captura. A Jaime Castillo y a todos los auxiliares de campo por su ayuda en esta fase. Al profesor Francisco Sánchez por sus valiosos comentarios de este escrito. Al señor José Jiménez por facilitar la estadía en su casa en inmediaciones del sector de Peña Amarilla durante algunas salidas de campo, así como a todos los habitantes del Parque por su buena disposición en la ejecución de este trabajo. Este trabajo formó parte del Programa de Investigación Ecología y Conservación de la Reserva Ranchería (Convenio 086-05 Corpoboyaca-UPTC).

\section{Literatura citada}

Albérico M, Cadena A, Hernández-Camacho J, Muñoz-Saba Y. 2000. Mamíferos (Synapsida: Theria) de Colombia. Biota Colomb. 1 (1): 43-75.

Anderson R. 1999. Preliminary review of the systematic and biogeography of the spiny pocket mice (Heteromys) of Colombia. Rev Acad Colomb Cienc. 23 (Suplemento especial): 613-30.

Anónimo. 2004. Creación del Parque Natural Municipal Ranchería de Paipa. Concejo Municipal de Paipa, Boyacá.

Barrera L, Vélez N. 2005. Plan de manejo para la Reserva Forestal Ranchería incluida su área de amortiguación. Municipio de Paipa, Jurisdicción de Corpoboyacá. Informe final. Contrato de consultoría, N 025 de 2004. $321 \mathrm{p}$.

Beer J. 1964. Bait preferences of some small mammals. $J$ Mammal. 45 (4): 632-34.

Cadena A, Malagón Z. 1994. Parámetros poblacionales de la fauna de pequeños mamíferos no voladores del cerro de Monserrate (Cordillera Oriental, Colombia). En: MoraOsejo LE, Sturm H (eds). Estudios ecológicos del páramo y bosque altoandino de la Cordillera Oriental de Colombia. Tomo II. Bogotá: Academia Colombiana Ciencias Exactas Física y Naturales, Colección Jorge Álvaro Lleras $\mathrm{N}^{\circ}$ 6. p. 538-618.

Carleton M, Musser G. 1989. Systematic studies of Oryzomyine rodents (Muridae, Sigmodontinae): a synopsis of Microryzomys. Bull Am Musem Nat Hist. 191: 1-83.

Corpoica. 2006. Informe final. Convenio de Cooperación Técnico-Económica $N^{\circ} 032$. Tunja: CORPOBOYACA.

Cuatrecasas J. 1958. Aspectos de la vegetación natural de Colombia. Rev Acad Colomb Cienc. 10 (40): 221-64.

Cujar A. 2005. Uso del hábitat del venado (Mazama rufina) en la Reserva Biológica de Cachalú y su área de influencia en los municipios del Encino y Charalá Santander. En: Solano C, Vargas N (eds). Memorias del I Simposio Internacional de Roble y Ecosistemas asociados. Bogotá: Fundación Natura-Pontificia Universidad Javeriana. p. 101-19.

Díaz J, Gómez-Laverde M. 2007. Comentarios sobre el primer registro de Micoureus alstoni (Allen 1990) (Didelphimorphia, Didelphidae) para Colombia. Actual Biol. 29 (87): 235-40.

Duran M, Kattan G. 2005. A test of the utility of exotic tree plantations for understory birds and foot resources in the Colombia Andes. Biotropica 37 (1): 129-35.

Etter A, McAlpine C, Wilson K, Phinn S, Possingham H. 2006. Patrones regionales de uso de la tierra agrícola y la deforestación en Colombia. Agricult, Ecosyst Environ. 114: $369-86$. 
Gardner AL. 2005. Order Didelphimorphia. En: Wilson E, Reeder M (eds). Mammal species of the World. $3^{\text {a }}$ ed. Washington, DC: Smithsonian Institution Press. p. 3-18.

Gómez-Laverde M. 1994. Los pequeños mamíferos no voladores del Parque Regional Natural Ucumarí. En: Rangel-Ch. JO (ed.). Ucumarí, un caso típico de la diversidad biótica andina. Pereira: Instituto de Ciencias Naturales (CARDER), Universidad Nacional de Colombia; p. 37796.

Gómez-Laverde M, Montenegro-Díaz O, López-Arévalo H, Cadena A, Bueno M. 1997. Karyology, morphology and ecology of Thomasomys laniger and T. niveipes (Rodentia) in Colombia. J Mammal. 78 (4): 12-82.

Gómez-Valencia B. 2004. Estructura de la comunidad de pequeños mamíferos en un gradiente altitudinal, reserva rio blanco, Manizales, Caldas, Colombia. (Trabajo de Grado). Bogotá, DC: Universidad Nacional de Colombia. $76 \mathrm{p}$.

Hernández J. 1993. Una sintesis de la historia evolutiva de la biodiversidad en Colombia. Nuestra diversidad biológica. Bogotá, DC: Fundación Alejandro Escobar.

Holdridge L. 1967. Life zone ecology. San José: Centro Científico Tropical; 206 p.

Ideam. 2008. Valores tomados de la estación metereológica La Rusia, Duitama-Boyacá de los años 1969 al 2007. Bogotá, DC: Ideam.

Linares J.O. 1998. Mamíferos de Venezuela. Caracas: Ed. Conservacionista Audubon de Venezuela; $691 \mathrm{p}$.

López N, Monroy M. 2003. Flórula arbustiva y arbórea de la Reserva Ranchería Paipa-Boyacá, Colombia. (Tesis de Grado). Tunja: Escuela de Ciencias Biológicas, Universidad Pedagógica y Tecnológica de Colombia.

López-Arévalo H, Montenegro-Díaz O. 1993. Mamíferos no voladores de Carpanta. En: Andrade GI (ed.). Carpanta: Selva nublada y páramo. Bogotá, DC: Fundación Natura. p. 165-87.

Nagorsen D, Peterson L. 1980. Mammal collector's manual: a guide for collecting documenting and preparing mammal specimens for scientific research. Toronto: Royal Ontario Museum.

Malagón Z. 1988. Estimación de algunos parámetros poblacionales de la fauna de pequeños mamíferos de la región de Monserrate. (Tesis de Grado). Bogotá, DC: Facultad de Ciencias, Universidad Nacional de Colombia. 93 p.

Montenegro-Díaz O, López-Arévalo H. 1990. Aspectos de la ecología y biología de pequeños mamíferos en una zona de bosque alto andino y páramo de la Reserva Biológica Carpanta. (Tesis Grado). Bogotá, DC: Universidad Nacional de Colombia. $164 \mathrm{p}$.

Musser G, Carleton D, Brothers E, Gardner A. 1998. Systematic studies of Oryzomyine rodents (Muridae, Sigmodontinae): diagnosis and distributions of species formerly assigned to Oryzomys "capito». Bull Am Museum Nat Hist. 236: 1-376.

Myers N. 1988. Threatened biotas: «hot spots» in tropical forests. Environment. 8 (3): 187-208.

Pardo M. 2007. Composición y estructura de la comunidad de murciélagos presente en el área de amortiguación del
Parque Natural Municipal Ranchería Paipa, BoyacáColombia. (Tesis de Grado). Tunja: Universidad Pedagógica y Tecnológica de Colombia; $107 \mathrm{p}$.

POT. 2000. Plan de Ordenamiento Territorial de Paipa. Ley $N^{\circ} 388$ de 1997. Proyecto de Acuerdo Municipal; Paipa (Boyacá, Colombia). Paipa: POT.

Rodríguez-Mahecha J, Albérico M, Trujillo F, Jorgenson J. 2006. Libro rojo de mamíferos de Colombia. Serie Libros Rojos de Especies Amenazadas de Colombia. Bogotá, DC: Conservación Internacional Colombia y Ministerio de Ambiente, Vivienda y Desarrollo Territorial. 433 p.

Sánchez F, Sánchez-Palomino P, Cadena A. 2004. Inventario de mamíferos en un bosque de los Andes centrales de Colombia. Caldasia. 26 (1): 291-309.

Voss R, Emmon L. 1996. Mammalian diversity in Neotropical lowland rainforests. A preliminary assessment. Bull Am Mus Nat Hist. 230: 1-115.

Voss R. 1988. Sistematics and ecology of Ichthiomyine (Muroidea) patterns morphological evolution in a small adaptive radiation. Bull Am Mus Nat Hist. 188: 259-493

Voss R. 1991. An introduction to the neotropical muroid rodent genus Zygodontomys. Bull Am Mus Nat Hist. 210: 113.

Voss R. 1992. A revision of the South American species of Sigmodon (Mammalia: Muridae) with notes on their natural history and biogeography. Bull Am Mus Nat Hist. 3050: 56.

Voss R, Lunde D, Simmons S. 2001. The mammals of Paracou, French Guiana: A neotropical lowland rainforest fauna. Part 2. Nonvolant species. Bull Am Mus Nat Hist. 263. 1-236.

Voss R, Jansa S. 2003. Phylogenetic studies on Didelphid Marsupials II. Non molecular data and new IRBP sequences: separate and combined analyses of Didelphine relationships with denser taxon sampling. Bull Am Mus Nat Hist. 276: 1-82.

Voss R, Gardner A, Jansa S. 2004. On the relationships of "Marmosa" formosa Shamel, 1930 (Marsupialia: Didelphidae), a phylogenetic puzzle from the Chaco of northern Argentina. Am Mus Novitates. 3442: 1-18.

Voss R, Lunde D, Jansa S. 2005. On the contents of Gracilinanus Gardner and Creighton, 1989, with the description of a previously unrecognized clade of small didelphid marsupials. Am Mus Novitates. 3482: 1-34.

Wilson E, Reeder M. 2005. Mammals species of the World: a taxonomic and geographic reference. Volume I. Washington, DC: Smithsonian Institution Press.

Woodman N. 2002. A new species of small-eared shrew from Colombia and Venezuela (Mammalia: Soricomorpha: Soricidae: Genus Cryptotis). Proceed Biol Soc. 115 (2): 249-72.

Woodman N, Timm R. 1993. Intraspecific and interspecific variation in the Cryptotis nigrescens species complex of small-eared shrews (Insectivora: Soricidae), with the description of a new species from Colombia. Zool Fieldiana. 74: 1-30.

Zúñiga H, Rodríguez J, Cadena A. 1990. Densidades de poblaciones de pequeños mamíferos de dos comunidades del bosque andino. Acta Biol Colomb. 1: 85-94. 